\title{
Reações Adversas aMedicamentose Farmacovigilância: Conhecimentos e Condutas de Profissionais de Saúde de um Hospital da Rede Sentinela
}

\section{Pharmacovigilance:Professional Knowledge and Conduct at a Teaching Hospital}

\author{
Ana Carolina Figueiredo Modesto \\ Tatyana Xavier Almeida Matteucci Ferreira \\ Mércia Pandolfo Provin ${ }^{I I}$ \\ Rita Goreti Amaral ${ }^{I I}$ \\ Dione Marçal Lima ${ }^{I I}$
}

\author{
PALAVRAS-CHAVE \\ - Conhecimentos, Atitudes e \\ Práticas em Saúde; \\ - Farmacovigilância; \\ - Reações Adversas a \\ Medicamentos; \\ - Educação Médica.
}

\section{KEYWORDS}

- Healthcare Knowledge;

- Attitudes, Practice;

- Pharmacovigilance;

- Adverse Drug Reaction Reporting Systems;

- Medical Education.

Introdução: As reações adversas a medicamentos são objeto de estudo da farmacovigilância, ciência que utiliza, sobretudo, as notificações espontâneas feitas por profissionais de saúde. Há dificuldade de reconhecimento dos profissionais quanto a sua importância na segurança do paciente. As causas da subnotificação são atribuídas a falta de conhecimentos, percepção e compreensão dos incidentes. Objetivo: Identificar o conhecimento e as condutas dos profissionais de saúde de um hospital de ensino em relação às reações adversas a medicamentos e à farmacovigilância e se há associação com o seu perfil profissiográfico. Métodos: Estudo transversal, utilizando-se um instrumento validado que aborda questões sobre caracterização da população estudada, conhecimentos e condutas. Resultados: Os médicos demonstraram maior conhecimento sobre reações adversas a medicamentos, e os técnicos em enfermagem, menor. No entanto, o entendimento sobre farmacovigilância foi maior entre os farmacêuticos. O tempo de formação e atuação na instituição está associado ao conhecimento sobre as reações adversas a medicamentos Conclusão: Os resultados deste estudo evidenciaram que a formação do profissional influencia seu conhecimento e condutas frente às reações adversas a medicamentos.

Introduction: Adverse Drug Reactions are studied by the science of pharmacovigilance, which makes particular use of spontaneous reports made by health professionals, although such professionals experience difficulties in recognizing adverse reactions and their importance in patient safety. The causes of underreporting are attributed to a lack of knowledge, awareness and understanding of incidents. Objective: Methods: A cross-sectional study was conducted using a validated instrument in order to characterize the population under study, determining its knowledge and behaviors Results: Doctors demonstrated a greater knowledge of adverse drug reactions than nursing technicians; however an understanding of pharmacovigilance was higher among pharmacists. The length of time spent in training and working in an institution were also associated with a knowledge of adverse drug reactions Conclusion: The results of this study show that professional training influences knowledge and behaviors on adverse drug reactions. 


\section{INTRODUÇÃO}

As reações adversas a medicamentos (RAM) são definidas como "qualquer resposta prejudicial ou indesejável e não intencional que ocorre com medicamentos em doses usualmente empregadas no homem para profilaxia, diagnóstico, tratamento de doença ou para modificação de funções fisio-

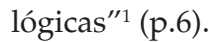

As RAM figuram entre a quarta e a sexta causa de mortes nos Estados Unidos², e no Brasil os estudos sobre o tema ainda são escassos. Além dos danos causados aos pacientes, existem custos associados ao tratamento das RAM, sendo que o paciente necessita de cuidados adicionais que resultam em impacto ao sistema de saúde, com a média de US\$ 3.332 por paciente ${ }^{3}$.

Inúmeros fatores podem influenciar no aparecimento de RAM, tais como idade, sexo, gênero, comorbidades e uso concomitante de vários medicamentos, entre outros. Alguns influenciam o aparecimento de RAM de maneira mais direta, e outros de forma insidiosa. Uma atenção maior dos profissionais de saúde pode auxiliar na sua identificação e prevenção4.

As RAM são objeto de estudo da farmacovigilância (FV), ciência que surgiu da necessidade de uma monitorização mais intensiva dos medicamentos após sua entrada no mercado, entre outras atividades relativas à Segurança do Paciente (SP) ${ }^{5}$.

Entre os diversos métodos utilizados na FV destaca-se a notificação espontânea (NE), efetuada pelos profissionais de saúde que lidam diretamente com a prescrição, dispensação e administração de medicamentos. Esse sistema proporciona um recurso efetivo para a identificação precoce de RAM raras e inesperadas, gerando sinais de alerta para os órgãos regulatórios. No entanto, o desafio a ser vencido é a dificuldade dos profissionais em reconheceras RAM e a importância de fazer a NE em sua rotina de trabalho. Estima-se que apenas $6 \%$ de todas as RAM sejam notificadas 6 .

As principais causas apontadas para a subnotificação são a falta de conhecimento sobre o que é uma RAM e seu impacto, a importância de notificar e como fazê-lo, a falta de tempo para preenchimento da documentação necessária, a falta de percepção e compreensão dos incidentes e o receio de punições ${ }^{7}$. Uma revisão sistemática demonstrou que os conhecimentos dos profissionais de saúde acerca das RAM e FV estão relacionados à prática de notificação ${ }^{8}$.

No contexto atual, a publicação da Portaria $n^{\circ} 529$, de $1^{\circ}$ de abril de 2013, que institui o Programa Nacional de Segurança do Paciente (PNSP), visa fortalecer a implementação do cuidado com os pacientes em todos os estabelecimentos de saúde 9 . Nesse sentido, a prática de notificação, que está contemplada entre as competências para a formação de profissionais voltados à segurança do paciente, deve ser encorajada entre os pro- fissionais, uma vez que a capacidade de analisar, sistematizar e aprender com as experiências se relaciona estreitamente com a cultura de segurança que deve permear as instituições de saúde, em especial as que trabalham diretamente com a prática de formação de novos profissionais dessa área ${ }^{10,11,12}$.

Este estudo identificou o conhecimento e as condutas dos profissionais de saúde de um hospital de ensino em relação às reações adversas a medicamentos e à farmacovigilância e se há associação com o seu perfil profissiográfico.

\section{MATERIAIS E MÉTODOS}

Este foi um estudo transversal, desenvolvido com os profissionais de saúde de uma unidade de internação de clínica médica e no serviço de farmácia de um hospital de ensino, inserido na rede sentinela, localizado no município de Goiânia, em Goiás, com coleta de dados realizada de outubro de 2013 a janeiro de 2014 .

Como critério de inclusão no estudo, estabeleceu-se a participação de profissionais que estavam diretamente relacionados aos processos de prescrição, dispensação e administração de medicamentos; seriam excluídos os profissionais que por motivo de férias ou licença de qualquer natureza não estavam exercendo suas atividades na clínica médica e serviço de farmácia durante o período da coleta de dados e aqueles que não se dispuseram a participar após terem sido contatados por três vezes consecutivas

De uma população de 229 profissionais, inseridos na clínica médica e no serviço de farmácia, 121 foram considerados elegíveis para participar do estudo. Destes, 76 concordaram em participar e assinaram o TCLE. No entanto, 22 não devolveram o instrumento de coleta de dados preenchido após três tentativas durante um período de 30 dias e foram considerados como perdas amostrais. A amostra final foi constituída por 54 profissionais de saúde.

O instrumento utilizado na coleta de dados foi adaptado de questionários empregados em pesquisas conduzidas com a mesma temática ${ }^{13,14,15}$. O questionário era constituído por três seções: a primeira compreendeu a caracterização demográfica e profissiográfica dos profissionais de saúde; a segunda e terceira seções continham questões com o objetivo de avaliar os conhecimentos e as condutas dos participantes em relação às reações adversas a medicamentos e à farmacovigilância, respectivamente.

As variáveis utilizadas para a caracterização do perfil profissiográfico dos profissionais de saúde foram: categoria profissional, tempo de formação, tempo de atuação na instituição, trabalhar em outro local, carga horária semanal total de trabalho; para o perfil demográfico, foram utilizados sexo e idade.

Para verificar o nível de conhecimento dos profissionais em relação às reações adversas a medicamentos e farmacovigi- 
lância, foram adotados os seguintes critérios: entendimento do conceito de reação adversa a medicamentos e farmacovigilância, conforme preconizado pela Organização Mundial da Saú$\mathrm{de}^{1,16}$; informações necessárias para efetuar uma notificação de reação adversa a medicamento, conforme orientações preconizadas pela Agência Nacional de Vigilância Sanitária ${ }^{17}$; quais reações adversas a medicamentos devem ser notificadas, bem como quais profissionais de saúde podem fazê-lo, conforme preconizado pela Organização Mundial da Saúde ${ }^{18}$; e os conhecimentos institucionais acerca do local que pode atuar frente às suspeitas de reações adversas a medicamentos e da inserção da instituição num programa nacional de farmacovigilância.

As condutas dos profissionais em relação às RAM foram verificadas por meio de questões que abordaram o que o profissional costuma fazer quando se depara com um caso suspeito de RAM. Para os médicos, as condutas incluíam: suspender o medicamento; modificar a terapêutica; ajustar a dose; manter o medicamento e tratar sinais e sintomas; não tomar nenhuma atitude. Para os enfermeiros e farmacêuticos, as condutas incluíam: registrar no prontuário e/ou comunicar ao médico; comunicar ao médico e suspender o medicamento; não tomar nenhuma atitude.

A análise estatística foi realizada de forma descritiva e também por meio do Teste Exato de Fischer e Regressão Logística. Os resultados foram apresentados por meio de frequência absoluta e relativa para a avaliação do perfil profissiográfico. Utilizou-se o Teste de Fischer e Regressão Logística para verificar se havia associação entre os conhecimentos e condutas dos profissionais de saúde em relação às RAM e à FV com seu perfil profissiográfico.

Este estudo foi aprovado pelo Comitê de Ética em Pesquisa do Hospital das Clínicas da Universidade Federal de Goiás, conforme protocolo ${ }^{\circ} 331.185$, de 10 de julho de 2013.

\section{RESULTADOS}

Participaram do estudo 54 profissionais de saúde das áreas de Medicina, Enfermagem, Farmácia e técnico em enfermagem. Observou-se que 79,6\% ( $n=43)$ eram profissionais do sexo feminino e 33,4\% ( $n=18)$ eram jovens, cuja faixa etária variou de 23 a 30 anos.

A Tabela 1 indica que, entre as categorias de profissionais, a maior participação foi dos técnicos em enfermagem, com $35,2 \%(n=19)$. Quase a metade dos participantes referiram um tempo inferior ou igual a cinco anos tanto para a conclusão do curso de formação, quanto para atuação deles na instituição, com $46,3 \%(n=25)$ e $48,1 \%(n=26)$, respectivamente. Mais da metade dos profissionais $(57,4 \%)$ declararam ter carga horária semanal total superior a 40 horas.

\begin{tabular}{|c|c|c|}
\hline \multicolumn{3}{|c|}{$\begin{array}{l}\text { Perfil profissiográfico dos profissionais de saúde inseridos } \\
\text { no estudo. Goiás, Brasil, } 2014 \text { (n = 54), Goiânia, } 2014\end{array}$} \\
\hline Variáveis & n & $\%$ \\
\hline \multicolumn{3}{|l|}{ Categoria profissional } \\
\hline Enfermeiro & 8 & 14,8 \\
\hline Farmacêutico & 11 & 20,4 \\
\hline Médico & 16 & 29,6 \\
\hline Técnico em enfermagem & 19 & 35,2 \\
\hline \multicolumn{3}{|c|}{ Tempo de conclusão do curso de formação } \\
\hline$\leq 5$ anos & 25 & 46,3 \\
\hline Entre 6 e 15 anos & 20 & 37,0 \\
\hline Entre 16 e 25 anos & 06 & 11,1 \\
\hline$\geq 26$ anos & 3 & 5,6 \\
\hline \multicolumn{3}{|c|}{ Tempo de atuação na instituição } \\
\hline$\leq 5$ anos & 26 & 48,1 \\
\hline Entre 6 e 15 anos & 21 & 38,9 \\
\hline Entre 16 e 25 anos & 7 & 13,0 \\
\hline$\geq 26$ anos & 0 & 0,0 \\
\hline \multicolumn{3}{|l|}{$\begin{array}{l}\text { Carga horária semanal } \\
\text { (incluindo todos os vínculos) }\end{array}$} \\
\hline$\leq 30$ horas & 17 & 31,4 \\
\hline Entre 31 e 40 horas & 3 & 5,5 \\
\hline Entre 41 e 60 horas & 31 & 57,4 \\
\hline$\geq 60$ horas & 3 & 5,5 \\
\hline
\end{tabular}

Os resultados referentes ao conhecimento sobre RAM e FV estão apresentados na Tabela 2. Em relação ao conhecimento sobre as RAM, 46,2\% dos participantes responderam corretamente. Os profissionais médicos apresentaram maior conhecimento sobre RAM quando comparados aos demais profissionais de saúde $(\mathrm{p}=0,00)$.

Quanto à compreensão sobre $\mathrm{FV}, 35,2 \%$ dos profissionais mostraram conhecimento sobre o conceito. Os farmacêuticos obtiveram o maior índice de acerto, com $47,3 \%$ relacionados a esta questão, e este índice mostrou-se significante em relação ao conhecimento demonstrado pelas demais categorias profissionais $(\mathrm{p}=0,00)$. $\mathrm{O}$ menor índice de acerto foi demonstrado pelos médicos $(p=0,01)$, dos quais apenas dois profissionais souberam responder corretamente.

Sobre o local da instituição que atua frente às suspeitas de RAM, bem como sobre a inserção da instituição num programa de FV, os farmacêuticos apresentaram maior conhecimento quando comparados com as demais categorias profissionais ( $p=0,00)$. Os médicos foram os que demonstraram menor conhecimento acerca da inserção desta instituição em algum programa de farmacovigilância $(p=0,00)$ (Tabela 2).

Observou-se associação entre tempo de trabalho do profissional na instituição e maior conhecimento sobre as RAM $(\mathrm{p}<0,01)$, conforme demonstrado no Gráfico 1. Igualmen- 


\begin{tabular}{|c|c|c|c|c|c|c|c|c|c|}
\hline \multicolumn{10}{|c|}{$\begin{array}{l}\text { TABELA } 2 \\
\text { issionais de saúde em relação às reações adversas } \\
\text { tos e farmacovigilância, Goiânia, } 2014\end{array}$} \\
\hline & \multicolumn{2}{|c|}{ Enfermeiro } & \multicolumn{2}{|c|}{ Farmacêutico } & \multicolumn{2}{|c|}{ Médico } & \multicolumn{2}{|c|}{ Técnico em enfermagem } & \multirow{2}{*}{$\begin{array}{l}\text { Total } \\
\text { n (\%) }\end{array}$} \\
\hline & n $(\%)$ & $\mathrm{p}$ & n (\%) & p & n (\%) & p & n (\%) & $\mathrm{p}$ & \\
\hline Conceito de RAM & $4(16,7)$ & 0,55 & $5(20,8)$ & 0,61 & $12(50,0)$ & $0,00^{*}$ & $3(12,5)$ & $0,00^{*}$ & $24(100)$ \\
\hline Conceito de FV & $3(15,8)$ & 0,60 & $9(47,3)$ & $0,00^{*}$ & $2(10,5)$ & $0,01^{*}$ & $5(26,4)$ & 0,28 & $19(100)$ \\
\hline $\begin{array}{l}\text { Setor responsável pela } \\
\text { atuação frente a RAM }\end{array}$ & $0(0,0)$ & 0,36 & $5(83,3)$ & $0,00^{*}$ & $0(0,0)$ & 0,16 & $1(16,7)$ & 0,40 & $6(100)$ \\
\hline $\begin{array}{l}\text { Inserção da instituição em } \\
\text { programa de FV }\end{array}$ & $5(23,8)$ & 0,23 & $10(47,6)$ & $0,00^{*}$ & $0(0,0)$ & $0,00^{*}$ & $6(28,6)$ & 0,30 & $21(100)$ \\
\hline
\end{tabular}

*Teste de Fischer

te, houve associação entre tempo de conclusão do curso de formação dos participantes e conhecimento sobre RAM ( $\mathrm{p}<$ 0,01 ) (Gráfico 2), sendo evidenciado que, quanto mais tempo o profissional de saúde tem de conclusão de sua formação e mais tempo de exercício profissional na instituição, maior seu conhecimento sobre RAM.

\section{GrÁfico 1}

Probabilidade de conhecimentos sobre reação adversa a medicamento em relação ao tempo de trabalho na instituição em anos

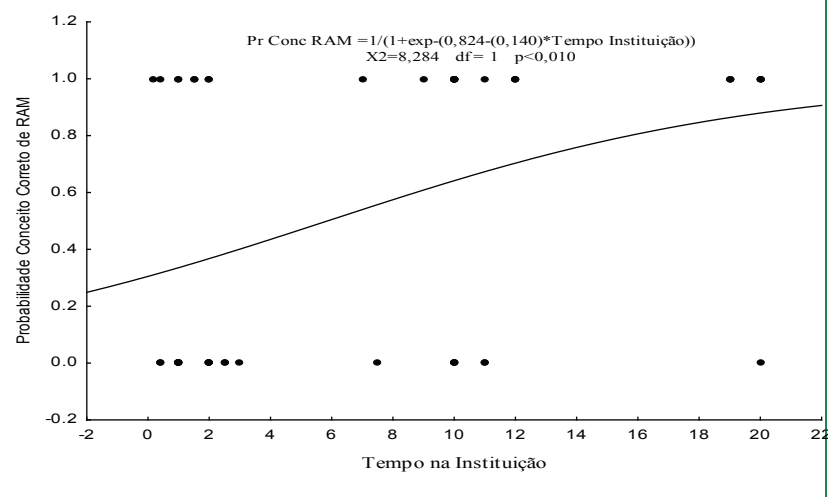

Não se observou associação entre conhecimento das atividades de FV e tempo de formação dos participantes, bem como tempo em que trabalham na instituição.

Em relação à conduta dos profissionais de saúde frente à RAM, observou-se que 53,7\% $(n=29)$ deles relataram ter conhecimentos sobre as informações necessárias para efetuar uma notificação de RAM no caso de se depararem com uma situação suspeita. Destes, os médicos foram os que mais informaram ter este conhecimento $(55,1 \%, n=16)$ quando compa- rados às demais categorias profissionais. Entretanto, quando questionados sobre como fazê-lo, apenas um farmacêutico fez menção correta do processo a ser percorrido para elaborar a notificação de uma RAM.

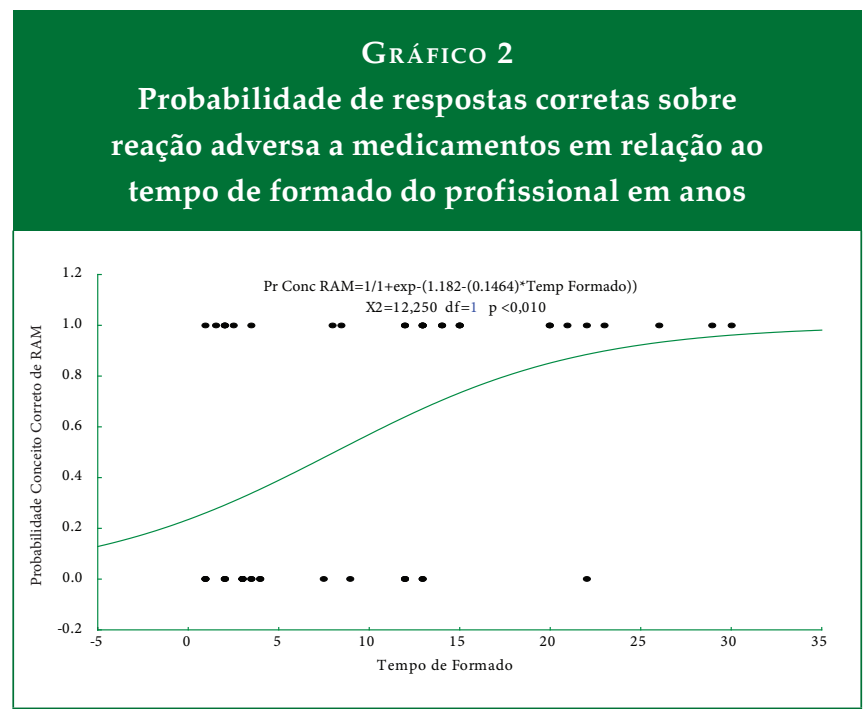

Na avaliação sobre quais RAM devem ser preferencialmente notificadas, $69,8 \%(\mathrm{n}=37)$ dos participantes informaram possuir este conhecimento; quanto a quais profissionais de saúde compete esta notificação de suspeitas de RAM, $94,4 \%$ ( $n=51)$ dos participantes souberam identificá-los corretamente.

Acerca das condutas dos participantes do estudo na prática diária de acompanhamento dos pacientes em uso de medicamentos, observou-se que 74,1\% $(n=40)$ declararam que ocasionalmente ou raramente se depararam com casos suspeitos de RAM no último ano, e 70,4\% relataram que sempre ou comumente questionam os pacientes sobre sinais e sintomas 
relacionados ao uso de medicamentos. Os técnicos em enfermagem foram a categoria que mais relatou esta atitude frente ao cuidado com os pacientes, com frequência de 39,5\% ( $n=15)$.

No que tange às dificuldades enfrentadas com relação à identificação de RAM, 52,4\% (n=44) relataram que o esquema terapêutico com administração simultânea de vários fármacos confunde o profissional e dificulta a identificação da RAM.

Os principais obstáculos que os profissionais de saúde relataram enfrentar para a realização das NE de RAM foram acúmulo de trabalho (34\%) e incerteza sobre o diagnóstico $(29,8 \%)$.

Os profissionais enfermeiros e farmacêuticos relataram que suas principais atitudes frente à suspeita de uma RAM são comunicar ao profissional médico e registrar a suspeita no prontuário do paciente; já os profissionais médicos relataram que a principal conduta adotada é suspender o uso do medicamento suspeito em $86,7 \%(n=13)$ das vezes.

Em relação à conduta adotada frente à segurança do paciente, foi observado que o fato de ter conhecimento sobre RAM não influencia as atitudes adotadas pelos profissionais enfermeiros e farmacêuticos (Tabela 3). No entanto, observou-se associação entre a conduta do médico de suspender o uso do medicamento suspeito e seu conhecimento sobre RAM $(p=0,00)$.

\begin{tabular}{|c|c|c|c|}
\hline \multicolumn{4}{|c|}{$\begin{array}{l}\text { Associação entre o conhecimento das RAM } \\
\text { demonstrado pelo profissional médico e suas } \\
\text { condutas práticas frente a RAM, Goiânia, } 2014\end{array}$} \\
\hline \multirow[t]{2}{*}{ Condutas práticas } & \multicolumn{3}{|c|}{$\begin{array}{c}\text { Conhecimento de RAM } \\
\text { pelos profissionais médicos }\end{array}$} \\
\hline & $\mathbf{n}$ & $\%$ & $\mathrm{p}$ \\
\hline Suspende o medicamento & 10 & 100,0 & $0,00^{*}$ \\
\hline Muda a terapêutica medicamentosa & 2 & 100,0 & $0,62^{*}$ \\
\hline Ajusta a dose & 0 & 0 & - \\
\hline $\begin{array}{l}\text { Mantém o medicamento trata sinais e } \\
\text { sintomas }\end{array}$ & 0 & 0 & - \\
\hline Não toma nenhuma atitude & 0 & 0 & - \\
\hline
\end{tabular}

*Teste de Fischer

\section{DISCUSSÃO}

Os resultados deste estudo sugerem que o conhecimento e as condutas acerca das RAM e FV estão associados à formação das diferentes categorias profissionais.

A compreensão sobre o conceito de RAM, embora superior à encontrada em outro estudo ${ }^{13}$, é baixa: pouco menos da metade dos entrevistados possuíam conhecimento básico sobre o tema. Entre as categorias profissionais, o médico foi quem apresentou maior conhecimento sobre as RAM, o que corrobora os achados de outros estudos similares ${ }^{13,19,20}$. Pode-se inferir que isto se deve à vivência diária deste profissional com o paciente, tendo no medicamento a principal ferramenta empregada como forma de tratamento no ambiente hospita$\operatorname{lar}^{21}$. Um médico forma suas competências para o cuidado com os pacientes aliando a teoria à prática, e nesse processo o internato e os estágios desempenham papel de grande importância, visto que possibilitam ao aluno a aplicação dos conhecimentos adquiridos, a incorporação de conhecimentos e a promoção do desenvolvimento de novas habilidades e atitudes que serão importantes para o seu futuro profissional ${ }^{22,23}$. Alguns estudos reportam uma taxa ${ }^{3} 30 \%$ de pacientes que sofrem algum tipo de RAM durante o seu tempo de internação ${ }^{2}$. Este fato, aliado à maior vivência deste profissional com o paciente e o medicamento durante o processo de formação desse profissional, pode torná-lo mais hábil a compreender e identificar as RAM.

A conduta do profissional médico de suspender o medicamento frente à suspeita de RAM é reportada em outro estudo em que em $83,6 \%$ dos casos esta foi a primeira atitude frente a um caso suspeito ${ }^{14}$, sendo considerada a conduta mais segura a $\operatorname{adotar}^{24}$. A suspensão de medicamentos é um rastreador de ocorrência de RAM, e essa associação do conhecimento influenciando diretamente a conduta adotada corrobora o fato de que a formação dessa categoria profissional se dá por meio de atividades práticas, geralmente sob supervisão de professor e/ou supervisor com experiência e vivência práticas, o que pode influenciar as condutas desses profissionais em formação ${ }^{23}$. Por outro lado, o profissional da área de enfermagem, que é parte importante da força de trabalho em ambiente hospitalar, além de ser responsável pelo preparo e administração de medicamentos, por ser o principal elo entre a equipe de saúde e o paciente, tem condições de comunicar ao médico assistente qualquer caso suspeito de RAM, e outros estudos corroboram essa conduta tomada pelo profissional de enfermagem $^{13,14,25}$.

No entanto, isto parece não ser suficiente para esse tipo de competência, pois, embora o enfermeiro e o técnico em enfermagem também possuam uma vivência intensa com os pacientes tanto durante sua formação quanto no exercício de suas atividades profissionais, o seu conhecimento sobre RAM foi baixo. A rigor, outras lacunas no processo de formação desses profissionais nessa área ${ }^{18,19}$ poderiam explicar este fato, tais como dificuldades na incorporação das propostas contidas nas Diretrizes Curriculares Nacionais (DCN) que versam sobre aquisição,desenvolvimento e avaliação das competências 
e habilidades necessárias ao desenvolvimento das atividades práticas desses profissionais ${ }^{26,27}$.

O pouco conhecimento sobre RAM pôde ser percebido por meio da dificuldade dos profissionais em reconhecer o local apropriado, seja em nível institucional ou nacional, para receber e processar as notificações de RAM, bem como as informações necessárias para preencher o formulário de notificação ${ }^{12,28}$. Ainda que este conhecimento seja baixo ${ }^{29,30}$, muitos profissionais reconhecem a necessidade de notificar todos os casos suspeitos de RAM, bem como a aptidão de qualquer profissional de saúde que trabalha diretamente na assistência para fazê-1o ${ }^{17,31}$.

Lacunas de conhecimento, pacientes necessitando de cuidados cada vez mais complexos e estrutura organizacional inadequada às atividades de notificação são dificuldades enfrentadas por parte dos profissionais de saúde no cotidiano das instituições de saúde. Sabe-se que 95\% das RAM manifestadas pelos pacientes ocorrem especialmente entre os que utilizam mais de cinco medicamentos, e esse perfil de paciente é comumente encontrado nas instituições hospitalares, visto que a média de medicamentos prescritos é de 5,8 por paciente, por dia ${ }^{19,32,33}$. Essas dificuldades podem ainda ser explicadas pelo fato de que existe uma complexa rede de variáveis consideradas como fatores desencadeantes de RAM no ambiente hospitalar; e a associação destes eventos com o conteúdo teórico abordado nos cursos de graduação em saúde pode não ter sido feita durante o processo de formação desse profissional, dificultando sua identificação e, em consequência disso, a notificação ${ }^{34,35}$. Há que se considerar ainda que a falta de recursos humanos sobrecarrega os profissionais em exercício, e, muitas vezes, o tempo que seria despendido nas atividades de notificação é voltado unicamente às atividades assistenciais que tais profissionais desempenham em suas diversas áreas de atuação ${ }^{36}$.

Estudos evidenciam a associação entre conhecimento dos profissionais de saúde e baixo índice de notificações de RAM, mostrando a importância da educação com a finalidade de promover o conhecimento e gerar mudanças de paradigmas nas atitudes desses profissionais frente às notificações, fazendo com que estes compreendam que tais atividades fazem parte de suas atribuições, além de serem importantes para a segurança dos pacientes sob seus cuidados ${ }^{7,30}$.

Diversos estudos têm mostrado a importância de estratégias educativas para o aumento das notificações de suspeitas de RAM nas instituições de saúde como parte de estratégias de educação permanente. Além disso, no presente estudo, foi evidenciada maior habilidade dos profissionais com maior vivência prática em conhecer as $\mathrm{RAM}^{29,37}$. No entanto, para que os profissionais recém-egressos dos cursos de graduação em saúde demonstrem conhecimentos satisfatórios acerca das RAM, é necessária uma reorganização na estrutura curricular dos cursos de modo a promover maior aproximação entre os estudantes e os cenários de prática, colocando em prática o currículo integrado. Desta forma, as instituições de saúde proporcionarão aos estudantes a articulação dos conteúdos abordados com sua aplicação prática, favorecendo uma aprendizagem significativa ${ }^{38,39,40}$.

Aliado ao baixo domínio conceitual, observou-se que mesmo aqueles que sabem o que é RAM não associaram sua ocorrência ao escopo das atividades da FV. Essa dissociação pode ser explicada pelo fato de que os profissionais de saúde desconhecem os conceitos e processos relativos às atividades de FV, entre os quais se inclui o preenchimento dos formulários de notificação ${ }^{29}$. Outros estudos mostraram que muitos profissionais não aderem aos programas de FV por acreditarem que os medicamentos utilizados na prática já foram devidamente testados em estudos clínicos e pré-clínicos e são totalmente seguros, além de desconhecerem a existência de sistemas de notificação, como evidenciado anteriormente ${ }^{41}$.

Essa dissociação da prática de notificação de RAM das atividades de FV demonstrada pelos médicos não foi observada entre os farmacêuticos, visto que essa categoria profissional, além de possuir extensa formação em farmacologia e toxicologia, relaciona a notificação de RAM como parte de suas obrigações profissionais, e existe ainda o fato de que notificações em âmbito hospitalar são comumente gerenciadas por esses profissionais 7,27 . Esses achados ainda podem ser vistos como um reflexo da formação dos profissionais de saúde no contexto do modelo biomédico, no qual, da mesma forma que esses profissionais enxergam os pacientes como partes de um todo, o mesmo pode ser feito com a FV, que não é encarada como ciência que se ocupa da monitorização e investigação da segurança dos medicamentos, que deve estar presente em todos os níveis de cuidado ${ }^{38}$.

O hiato entre o saber e o fazer pode ser reflexo do distanciamento entre a teoria e a prática vivenciado nas instituições de saúde brasileiras. Um estudo conduzido com alunos de graduação de Medicina e Enfermagem em universidade pública de São Paulo mostrou que quase a totalidade dos alunos $(91,6 \%)$ relatou que existe uma grande diferença entre o que os profissionais de saúde sabem e o que de fato é observado no dia a dia da assistência, e esse resultado pode estar associado com a dificuldade de assimilação dos conteúdos teóricos, pela inexistência de correlação destes com a prática ${ }^{35}$.

A predominância de profissionais do sexo feminino no presente estudo também foi identificada por outros autores em pesquisas conduzidas em hospitais de ensino ${ }^{13,31}$. Estudos 
têm demonstrado que as mulheres representam maior número em acesso às universidades e formação superior especialmente nos cursos da área da saúde, o que pode ser evidenciado pela mudança no perfil dos egressos do curso de Medicina, onde o número de mulheres tem aumentado significativamente nas últimas décadas ${ }^{42,43,44}$. A maior prevalência encontrada no presente estudo pode estar relacionada também a maior percentagem de participantes da área de formação técnica e superior da área de Enfermagem, onde a grande maioria é representada por indivíduos do sexo feminino ${ }^{45,46}$.

A predominância de profissionais com faixa etária mais jovem e com pouco tempo de conclusão do curso de formação, no presente estudo, contrasta com os resultados encontrados por outros autores em pesquisas conduzidas em hospitais ${ }^{13,47}$. Essas diferenças apontadas possivelmente ocorreram pelo fato de que o presente estudo foi conduzido num hospital de ensino, onde são encontrados vários profissionais que ainda se encontram em processo de formação, como nos programas de residência multiprofissional e médica, recém-egressos de cursos de graduação da área da saúde.

A maior representatividade de profissionais da área de Enfermagem pode ser justificada pelo fato de que a equipe de enfermagem é encontrada em maior número nos estudos de serviços hospitalares, onde comumente ocupam importante espaço no grupo de recursos humanos ${ }^{48}$. Este resultado também foi verificado em outro estudo realizado com a população presente no ambiente hospitalar ${ }^{8}$.

A FV é uma ciência consolidada em países desenvolvidos, que inclui aspectos relativos à segurança do paciente. Nas instituições de saúde, ainda é incipiente em países da América Latina, incluindo o Brasil, que dispõe de legislações que regulamentam essas atividades. A notificação, não só de RAM, mas de qualquer Evento Adverso (EA) passou a ser compulsória em estabelecimentos de saúde de todo o território nacional após a publicação da portaria que instituiu o Programa Nacional de Segurança do Paciente (PNSP) ${ }^{9,29}$.

As notificações, como principal método utilizado na FV, ainda desempenham importante papel no processo de mudança do paradigma da cultura punitiva, ainda presente nas instituições de saúde, para a cultura de segurança, na qual os profissionais de saúde são estimulados a relatar incidentes decorrentes da prática assistencial com o objetivo de refletir e aprender com eles ${ }^{49}$.

Há um caminho longo a percorrer, mas sem volta, e há que se refletir que as competências do profissional de saúde relacionadas às atividades de FV devem ser construídas no decorrer da sua formação e consolidadas na prática profissional, uma vez que os conhecimentos e habilidades adquiridos du- rante a graduação se complementam com as atitudes práticas requeridas durante os estágios, bem como na vivência diária. É necessário reconhecer essas fragilidades e trabalhar no sentido de seguir rumo a uma profunda reorientação nos processos de ensino-aprendizagem voltados à segurança dos pacientes nas instituições ${ }^{12,50}$.

\section{CONCLUSÕES}

Os resultados deste estudo demonstram que existe uma lacuna entre o conhecimento teórico apreendido pelos profissionais de saúde no decorrer dos cursos de formação, sejam estes de nível médio ou superior, e as atitudes práticas, materializadas pela falta de reconhecimento da importância da identificação das RAM experimentadas pelos pacientes e sua notificação aos órgãos regulatórios com vistas a promover uma utilização segura e racional dos medicamentos, uma vez que estes constituem a principal ferramenta terapêutica utilizada nas instituições de saúde.

O fato de os profissionais médicos e farmacêuticos terem demonstrado conhecimentos somente no âmbito de suas áreas específicas reflete a necessidade de estímulo ao trabalho interdisciplinar e multiprofissional, para que cada um, na sua área, possa contribuir para a construção de uma rede colaborativa para a promoção de uma vigilância ativa da segurança dos medicamentos.

Preocupa o fato de os profissionais da área técnica em enfermagem, que constituem a grande maioria no âmbito das instituições de saúde, contribuindo com a primeira linha de cuidado junto aos pacientes, terem demonstrado tão pouco conhecimento acerca das RAM e sua importância para a segurança dos pacientes.

Os resultados deste estudo poderão contribuir com subsídios para o planejamento de estratégias de sensibilização a respeito da importância das atividades de FV para a identificação das RAM e a promoção do uso racional de medicamentos voltados ao cuidado do paciente.

Outras estratégias devem ser discutidas, e todas as instituições formadoras de recursos humanos para a saúde devem repensar seus currículos e promover maior integração ensino-serviço. Por outro lado, as instituições de saúde devem investir em estratégias educativas dos profissionais inseridos no mercado de trabalho para a preparação de profissionais aptos para identificação de situações de risco, discussão de condutas em equipe, notificação de incidentes,entre outras, que são necessárias à consolidação da cultura de segurança que deve permear as instituições de saúde, especialmente as formadoras de recursos humanos para a saúde, conforme preconizado pela Organização Mundial de Saúde. 


\section{REFERÊNCIAS}

1. Organização Mundial de Saúde. Segurança dos medicamentos: um guia para detectar e notificações de reações adversas a medicamentos. Por que os profissionais de saúde precisam entrar em ação. Organização Mundial de Saúde [on line]. 2005. [capturado 10 out. 2014]; 18. Disponível em: http:/ /www.sbrafh.org.br/site/index/library/ $\mathrm{id} / 55$.

2. Beijer HJ, Blaey CJ. Hospitalisations caused by adverse drug reactions: a meta-analysis of observational studies. Pharm World Sci. 2002;24(2):46-54.

3. Vallano FA, Agustí EA, Pedrós XC, Arnau BJM. Systematic review of studies assessing the cost of adverse drug reactions. Gac Sanit [on line]. 2012. 26(3) [capturado 01 out. 2014]; 277-83. Disponível em: http://www.ncbi.nlm.nih. gov/pubmed/22152925.

4. Alomar MJ. Factors affecting the development of adverse drug reactions. Saudi Pharm J [on line]. 2014. 22(2) [capturado 01 out. 2014];83-94. Disponível em: http://dx.doi. org/10.1016/j.jsps.2013.02.003.

5. Varallo FR, Mastroianni PC. Farmacovigilância:avaliação do risco/benefício para a promoção do uso seguro de medicamentos. In: Mastroianni PC, Varallo FR, org. Farmacovigilância para promoção do uso correto de medicamentos. Porto Alegre: Artmed; 2013. p. 13-26.

6. Hazell L, Shakir SA. Under-reporting of adverse drug reactions: a systematic review. Drug Saf. 2006;29(5):385-96.

7. Primo LP, Capucho helaine C. Intervenções educativas para estímulo a notificações voluntárias em um hospital de ensino da rede sentinela. Rev Bras Farmácia Hosp e Serviços Saúde. 2011;2(2):26-30.

8. Lopez-Gonzales E, Herdeiro MT, Figueiras A. Determinants of under-reporting of adverse drug reactions: a systematic review. Drug Saf. 2009;32(1):19-31.

9. Brasil. Ministério da Saúde. Portaria n. 529, de 1 de abril de 2013. Institui o programa nacional de segurança do paciente. Diário Oficial da União. Brasília, 2 abr. 2013; Seção 1, p. 43.

10. Reis T. A segurança do paciente como dimensão da qualidade do cuidado de saúde - um olhar sobre a literatura. Cien Saude Colet. 2013;18(7):2029-36.

11. Corbellini VL, Schilling MCL, Frantz SF, Godinho TG, Urbanetto J de S. Eventos adversos relacionados a medicamentos: percepção de técnicos e auxiliares de enfermagem. Rev Bras Enferm [on line].2011. [capturado 27 nov. 2014];64(2):241-7. Disponível em: http://www.scielo.br/ scielo.php?script=sci_arttext\&pid=S0034716720110002000 $04 \& \operatorname{lng}=\mathrm{en} \& \mathrm{nrm}=\mathrm{iso} \& \ln \mathrm{g}=\mathrm{pt}$
12. Urbanetto JDS, Gerhardt LM. Segurança do paciente na tríade assistência ensino pesquisa. Rev Gaúcha Enferm [capturado 01 out. 2014]. 2013;34(3):8-9. Disponível em: http:/ / www.scielo.br/scielo.php?script=sci_arttext\&pid $=$ S198314472013000300001\&lng $=$ en\&nrm $=$ iso\&tlng $=$ pt.

13. Pinheiro HCG, Vera Lúcia EP. Reações adversas a medicamentos : conhecimento e atitudes dos profissionais de saúde em um hospital- sentinela de ensino do Ceará-Brasil. 2008. Epidemiol e Serviços Saúde. 2011;20(1):57-64.

14. Salviano LHMS, Luiza VL, Ponciano AMDS. Percepção e condutas de profissionais da Estratégia Saúde da Família acerca de reações adversas a medicamentos. Epidemiol e Serviços Saúde. 2011 Mar;20(1):47-56.

15. Carvalho JP. Estratégias para a elaboração do plano de farmacovigilância considerando o conhecimento e opinião dos profissionais de uma indústria pública. Rio de Janeiro; 2011. Mestrado [Dissertação] - Escola Nacional de Saúde Pública Sérgio Arouca.

16. Organização Pan-Americana da Saúde. A importância da farmacovigilância. Monitorização da segurança dos medicamentos [on line].2005. [capturado 10 out. 2014];48.Disponível em: http://bvsms.saude.gov.br/bvs/publicacoes/ importancia.pdf.

17. Agencia Nacional de Vigilância Sanitária. Sistema Nacional de Notificações para a Vigilância Sanitária-Manual do usuário; Anexo 3-Formulário para Notificação de Eventos Adversos à Medicamento-Profissional da Saúde [on line]. 2011; [capturado 10 out. 2014];39. Disponível em: http:// www.anvisa.gov.br/hotsite/notivisa/manual/ea_medicamento_profissional.pdf.

18. Organisation Mondiale de la Santé. Surveillance de la sécurité d'emploi des médicaments.Uppsala, 2000. (Relatório)

19. Rehan HS, Sah RK, Chopra D. Comparison of knowledge, attitude and practices of resident doctors and nurses on adverse drug reaction monitoring and reporting in a tertiary care hospital. Indian J Pharmacol. 2014;44(6):699-703.

20. Rehan HS, Chopra D, Sah RK, Mishra R. Adverse Drug Reactions : Trends in a Tertiary Care Hospital. Curr Drug Saf. 2012;7:384-8.

21. Pirmohammed M, Meakin JM. Adverse drug reactions as cause of admission to hospital: prospective analysis of 18.820 patients. Br Med J. 2004;24:46-54.

22. Franco CAGS, Cubas MR, Franco RS. Curriculo de medicina e as competências propostas pelas diretrizes curriculares. Rev Bras Educ Med. 2014;38(2):221-30.

23. Campos MCG, Senger MH. Avaliação do estágio de urgências clínicas em uma unidade de pronto atendimen- 
to sob a perspectiva dos alunos. Rev Bras Educ Med. 2011;38(1):103-12.

24. Edwards IR, Aronson JK. Adverse drug reactions: definitions, diagnosis, and management. Lancet. 2000; 356(7): 1255-9.

25. Telles Filho PCP, Cassiani SHB. Administração de medicamentos: aquisição de conhecimentos e habilidades requeridas por um grupo de enfermeiros. Rev Lat Am Enferm. 2004;12(3):533-40.

26. Marran AL, Lima PG, Bagnato MHS. As políticas educacionais e o estágio curricular supervisionado no curso de graduação em enfermagem. Trab Educ e Saúde. 2015;13(1):89-108.

27. Colenci R, Berti W. Formação profissional e inserção no mercado de trabalho: percepções de egressos de graduação em enfermagem. Rev da Esc Enferm. 2012;46(1):158-66.

28. Ahmad A, Patel I, Balkrishnan, Mohanta, G P, Manna PK. An evaluation of knowledge, attitude and practice of Indian pharmacists towards adverse drug reaction reporting: A pilot study. Perspect Clin Res. 2013;4(4):204-10.

29. Varallo FR, Guimarães SDOP, Abjaude SAR, Mastroianni PDC. Causes for the underreporting of adverse drug events by health professionals: a systematic review. Rev da Esc Enferm da USP [on line]. 2014;48(4):739-47.[capturado 01 out. 2014]. Disponível em: http:/ /www.scielo.br/scielo.php?script=sci_arttext\&pid=S008062342014000400739\& $\operatorname{lng}=\mathrm{en} \& n r m=$ iso\&tlng=en

30. Oshikoya KA, Awobusuyi JO. Perceptions of doctors to adverse drug reaction reporting in a teaching hospital in Lagos, Nigeria. BMC Clin Pharmacol. 2009;9(14):1-8.

31. Palaian S, Ibrahim MI, Mishra P. Health professionals ' knowledge, attitude and practices towards pharmacovigilance in Nepal. Pharm Pract. 2011;9(4):228-35.

32. Néri EDR, Gadelha PGC, Maia SG, Pereira AG da S, Almeida PC, Rodrigues CRM, et al. Erros de prescrição de medicamentos em um hospital brasileiro. Rev Assoc Medica Bras. 2011;57(3):306-14.

33. Araújo PTB, Uchôa SAC. Avaliação da qualidade da prescrição de medicamentos de um hospital de ensino. Cien Saude Colet. 2011;16(Suplemento 1):1107-14.

34. Ferner RE, Aronson J. Preventability of drug-related harms Part I: A Systematic Review. Drug Saf. 2010;33(11):985-94.

35. Yoshikawa JM, Sousa BEC, Peterlini MAS, Kushara DM, Pedreira MLG, Avelar AFM. Compreensão de alunos de graduação em enfermagem e medicina sobre segurança do paciente. Acta Paul Enferm. 2013;26(1):21-9.

36. Sodré F, Littike D, Drago LMB, Perim MCM. Empresa brasileira de serviços hospitalares: um novo modelo de gestão? Serviço Soc e Soc. 2013;2(114):365-80.
37. Pagotto C, Varallo F, Mastroianni P. Impact of educational interventions on adverse drug events reporting. Int $\mathrm{J}$ Technol Assess Health Care [on line]. 2013 Oct [Capturado 27 nov. 2014];29(4):410-7.Disponível em: http://www.ncbi. nlm.nih.gov/pubmed/24290334.

38. Araújo D, Miranda MCG, Brasil S. Formação de profissionais de saúde na perspectiva da integralidade. Rev Baiana Saúde Pública. 2007;38(4):511-7.

39. Cotta RMM, Reis RS, Campos AA de O, Gomes AP, Antonio VE, Siqueira-Batista R. Debates atuais em humanização e saúde: quem somos nós? Cien Saude Colet. 2013;18(1):171-9.

40. Almeida MM, Morais RP, Guimarães DF, Machado MFAS, Nuto SAS. Experiência do Pró-Saúde Unifor e Seus Nove Cursos de Graduação. Rev Bras Educ Med. 2010;36(1):119_ 26.

41. Khalili H, Mohebbi N, Hendoiee N, Keshtkar A. Improvement of knowledge, attitude and perception of healthcare workers about Adverse Drug Reactions, a pre-and post-clinical pharmacists' interventional study. Br Med J. 2012;2(10):1-5.

42. Castellanos MEP, Fagundes T de LQ, Nunes TCM, Gil CRR, Pinto ICM, Belisário SA, Viana SV, Correa, GT, Aguiar, RAT. Estudantes de graduação em saúde coletiva-perfil sociodemográfico e motivações. Ciência. 2013;18(6):165766.

43. Ávila RC. Formação das Mulheres nas Escolas de Medicina. Rev Bras Educ Med. 2014;38(1):142-9.

44. John LJ, Arifulla M, Cheriathu JJ, Sreedharan J. Reporting of adverse drug reactions : an exploratory study among nurses in a teaching hospital,Ajman, United Arab Emirates. DARU J Pharm Sci. 2012;20(1):1-6.

45. Alves PC, Neves VF, Ferreira M, Coleta D. Avaliação do bem-estar no trabalho entre profissionais de enfermagem de um hospital universitário. Rev Lat Am Enferm. 2012;20(4):1-8.

46. Dos Santos Pernas SI, Herdeiro MT, Lopez-Gonzalez E, da Cruz e Silva OB, Figueiras A. Attitudes of Portuguese health professionals toward adverse drug reaction reporting. Int J Clin Pharm [on line]. 2012. 34(5) [capturado em 1 oct. 2014];693-8. Disponível em: http://www.ncbi.nlm.nih. gov/pubmed/22936287.

47. Almeida ML, Segui MLH, Bernardino E, Méier MJ, Peres AM. Direção ou Coordenação?Repensando a representatividade institucional da Enfermagem. Rev Bras Enferm. 2011;64(3):521-6.

48. Alshakka MA, Izham M, Ibrahim M, Azmi M, Hassali A. Do Health Professionals have Positive Perception Towards 
Consumer Reporting of Adverse Drug Reactions? J Clin Diagnostic Res. 2013;7(10):2181-5.

49. Capucho HC, Arnas ER, Cassiani SH de B. Segurança do paciente: comparação entre notificações voluntárias manuscritas e informatizadas sobre incidentes em saúde. Rev Gauch Enferm. 2013;34(1):164-72.

50. Rosa K, Beatriz S, Krug F, Garcia EL. Práticas interdisciplinares no programa de educação pelo trabalho para a saúde e vigilância em saúde : contribuições para a formação do profissional farmacêutico. Rev Epidemiol e Control infecção. 2014;4(4):2-5.

\section{CONTRIBUIÇÃO DOS AUTORES}

Ana Carolina Figueiredo Modesto: elaboração do projeto, coleta de dados, análise de dados e redação do artigo. Tatyana Xavier Almeida Matteucci Ferreira: elaboração do projeto, análise de dados e redação do artigo. Mércia Pandolfo Provin, Rita Goreti Amaral e Dione Marçal Lima: elaboração do projeto; redação do artigo e revisão final do texto.

\section{CONFLITO DE INTERESSES}

Os autores declaram não haver conflito de interesses.

\section{ENDEREÇO PARA CORRESPONDÊNCIA}

Ana Carolina Figueiredo Modesto

Primeira Avenida, s/n

Bairro: Setor Leste Universitário - Goiânia

CEP: 75.605-020 GO

E-mail: farmcarolina@gmail.com 